Methods 228 patients with acute coronary syndrome undergoing delayed percutaneous coronary intervention were randomly divided into standard statin group (SSG $n=115$ ) and intensive statin group (ISG $\mathrm{n}=113$ ). Patients in SSG were given simvastatin $20 \mathrm{mg}$ /day and patients in ISG were given simvastatin $80 \mathrm{mg}$ /day for at least 7 days before PCI, Serum creatinine was measured at admission, $24 \mathrm{~h}$ and $48 \mathrm{~h}$ after PCI, and the Creatinine clearance was calculated. The levels of hs-CRP, ICAM-1 and P-selectin were also measured.

Results Serum creatinine underwent significant increase after PCI, the peak value occurred at $24 \mathrm{~h}$, and then began to decrease. At $48 \mathrm{~h}$ after PCI, the creatinine level significantly decreased $(p<0.001)$ to baseline level in ISG, whereas in SSG the creatinine level failed to decrease significantly. Serum creatinine at admission was not significantly different between the two groups, But at 24th and 48th hour after PCI, it were lower in ISG than SSG $(p<0.05$ at 24 th hour and $\mathrm{p}<0.001$ at 48 th hour). The creatinine clearance significantly decreased after PCI, the lowest value occurred at $24 \mathrm{~h}$, and then it began to increase. In SSG, the creatinine clearance increased significantly $(p=0.03)$ at $48 \mathrm{~h}$, but still significantly lower than baseline level. In ISG, the creatinine clearance increased significantly $(p<0.001)$ at $48 \mathrm{~h}$ and recovered to the level at baseline. Creatinine clearance improved much more in ISG at 24 and $48 \mathrm{~h}$ than that in SSG ( $p<0.001$ at 24 th hour and at 48th hour). Although procedure caused significant increase in hs-CRP, P-selectin and ICAM-1 $(p<0.001)$, the increase in ISG was smaller than SSG $(p<0.001)$.

Conclusion Pretreatment with intensive statin dosage before PCI can further decrease the occurrence of CIN. This benefit may be associated with the lowering of hs-CRP, P-selectin and ICAM levels.

\section{e0647 THE ANIMAL MODEL ESTABLISHMENT OF ACUTE CORONARY NO-REFLOW PHENOMENON IN YORK SWINE VIA INTRACORONARY INJECTION OF MICROSPHERE WITH BLOOD SUSPENSION}

doi:10.1136/hrt.2010.208967.647

Fu Xianghua, Fan Weize, Gu Xinshun, Wang Yanbo, Wang Xuechao, Hao Guozhen. The 2nd Hospital of Hebei Medical University, Shijiazhuang, Hebei, China

Objective To explore the fusibility of establishing animal model of no reflow phenomenon in swine by super selecting LAD with $4 \mathrm{~F}$ catheter, and injecting polyethylene microspheres and blood suspension (PMBS) into LAD.

Methods Total of 15 york swines were included in this study. CAG was performed by $4 \mathrm{~F}$ micro-catheter technique, and PMBS was injected into the superselected LAD. The coronay blood flow was evaluated by TIMI frame counts and the level of myocardial tissues perfusion was evaluated by TMPG. The model of NRP of AMI was considered as success while TIMI blood flow being less than grade 2 or TFC more than 36.2 counts or TMPG less than grade 1 . Left ventriculography was performed with 4F Pigtail and left ventricular systolic, diastolic pressure was recorded. MRAP, mRVP, mPAP and PCWP were measured by swan-ganz floating catheter, and CO was measured by thermodilution. ECG and blood pressure were monitored, and platelet aggregate ratio, CK-MB, TnI and blood gas analysis were measured. Ischaemic region, normal region and borderline were scissored respectively and sent to check for pathology, and the necrotic zone of myocardium was weighed and the percentage of which in left ventricle was calculated.

Results According to the standards of NRP, 11 animals achieved the NRP model of AMI successfully, achievement ratio of the models was $73.3 \%$, average times of injection of PMBS were $3.2 \pm 0.6$. While the model of NRP was established, instant ECG showed that ST segment elevated and formed single-direction curve with high $\mathrm{T}$ wave, and $\mathrm{R}$ wave was gradually depressed. Though $\mathrm{PO}_{2}$ was decreased after establishment of NRP model, the level of which was in normal range. PAP, PCWP and LVEDP were increased at instantly, 30, 60, 120,
180 min after NRP with AMI, which had statistical significance $(p<0.05)$. SBP and DBP were decreased after successful establishment of NRP model. Heart rate was increased $(p<0.05)$ and LVP was decreased $(p<0.05)$ after successful establishment of NRP model. Decreasement of mean perfusion pressure was negative correlation with TFCs. Pathological examination showed that myocardium fibre swelling, sarcolysis, reticular formation and local liquefaction necrosis occurred. The area of myocardiolysis in the left ventricle was $28.6 \%$. Conclusion This model had advantages of direct-viewing, simplicity, repetitiveness, mild trauma, closed chest, high achievement ratio and more similar to NRP after PCI in clinical compared with myocardium dyeing and other methods. It might provide better experimental animal model for microcirculation disturbance after AMI.

\section{e0648 EFFECTS OF PRETREATMENT WITH SIMVASTATIN ON THE AREA OF MYOCARDIAL INFARCTION IN REPERFUSION INJURY RABBITS AFTER ACUTE MYOCARDIAL INFARCTION}

doi:10.1136/hrt.2010.208967.648

Fu Xianghua, Ding Chao, Fan Weize, Gu Xinshun, Wu Weili, Li Shiqiang, Hao Guozhen, Jiang Yunfa. The 2nd Hospital of Hebei Medical University, Shijiazhuang, Hebei, China

Objective To investigate the effects and mechanism of the pretreatment with simvastatin on the area of myocardial infarction in reperfusion injury rabbits after acute myocardial infarction (AMI).

Method $20 \mathrm{New}$ Zealand white rabbits were randomly divided into four groups: group A, AMI/reperfusion; group B, pretreated with simvastatin ( $5 \mathrm{mg} / \mathrm{kg}$ ) for 3 days before AM I; group C, treated with glibenclamide (K $\mathrm{K}_{\text {ATP }}$ channel blocker) $(5 \mathrm{mg} / \mathrm{kg}$ ) before AMI, group $\mathrm{D}$, treated with glibenclamide and simvastatin before AMI. Models of $\mathrm{AMI} /$ reperfusion were established by 180 -min of coronary occlusion and $60-\mathrm{min}$ of reperfusion. At the end of reperfusion, the coronary artery was reoccluded, and the risk zone was delineated with Evan' blue. Hearts were sectioned $(2 \mathrm{~mm})$ and incubated in $1 \%$ TTC in phosphate buffer for 20 min to define white necrotic tissue when fixed in $10 \%$ formalin for $24 \mathrm{~h}$, and the level of plasma creatine kinase-MB was assessed and evaluated.

Result (1)The content of CK-MB was significantly decreased in group $B$ than that in group $A$ and group $C(P<0.01)$, however, it was markedly decreased in group $D$ than those of group $A$, and significantly increased than that of group B. (2) The risk zone volumes were similar among all the groups. The infarct size was (43.6 \pm 4.6$) \%$ in group A, Simvastatin treatment resulted in a significant limitation of infarct size in group $\mathrm{B}(23.6 \pm 2.8 \%$ VS group $\mathrm{A}, \mathrm{P}<0.01)$, the infarct size was similar in group $\mathrm{C}(45.1 \pm 4.5 \%)$ compared with that in group $\mathrm{A}(\mathrm{P}>0.05)$. However, it was markedly decreased in group $\mathrm{D}(36.8 \pm 3.4 \%)$ than that of group $\mathrm{A}(\mathrm{P}<0.05)$, and significantly increased than that of group $\mathrm{B}(\mathrm{P}<0.05)$.

Conclusion Simvastatin significantly reduced myocardial infarct size and the level of plasma myocardial enzyme during AMI and reperfusion in rabbits, which has protective effects on ischaemic/reperfused myocardium, and the activation of ATP-sensitive K channels might be involved in this protective mechanism.

\section{e0649 LONG-TERM EFFECTS OF RA-URIS PACING ON LEFT VENTRICULAR REMODELLING IN PATIENTS WITH CHRONIC HEART DYSFUNCTION}

doi:10.1136/hrt.2010.208967.649

Fu Xianghua, Ma Ning, Liu Xiaokun, Li Luming, Wang Xuechao, Wang Yanbo. The 2nd Hospital of Hebei Medical University, Shijiazhuang, Hebei, China

Objective To assess the effects of RA-URIS dual chamber sequential pacing on left ventricular remodelling and cardiac function in SSS or AVB patients with chronic heart dysfunction. 